\title{
Interface properties influence the effective dielectric constant of composites
}

\author{
G. Chatzigeorgiou ${ }^{\mathrm{a}}$, A. Javili ${ }^{\mathrm{b} *}$ and P. Steinmann ${ }^{\mathrm{c}}$ \\ ${ }^{a}$ LEM3-UMR 7239 CNRS, Arts et Métiers ParisTech, 4 Rue Augustin Fresnel, 57078 Metz, France; \\ ${ }^{b}$ Department of Civil and Environmental Engineering, Stanford University, 473 Via Ortega, \\ Stanford, CA 94305, USA; ${ }^{c}$ Applied Mechanics, University of Erlangen-Nuremberg, Egerlandstr. 5, \\ 91058 Erlangen, Germany
}

\begin{abstract}
In this work we propose a micromechanics model, based on the composite spheres assemblage method, for studying the electrostatic behaviour of particle and porous composites when the interface between the particle (or the pore) and the matrix has its own independent behaviour. Examples from experimental results in porous media and particle composites are utilized to demonstrate the model's capabilities. The current model shows a better agreement with experiments compared with other available studies. Furthermore, it is shown that the interface dielectric constant is independent of the experiments which prove its nature as a material parameter.
\end{abstract}

\section{Introduction}

The interfaces between particles and matrix material play a significant role in the relative permittivity [1-3]. It has been shown experimentally that the electric performance of a particle can be improved with surface modification [4-7]. It has also been observed that the increase in the area-to-volume ratio (i.e. decrease in the particle and/or grain size) can influence substantially the dielectric constant [8,9]. McCarthy et al. [10] investigate the importance of interfacial interactions between the nanoparticles and the matrix for dielectric materials. Related works include e.g. [11] which detail the improvement of electrostrictive properties of elastomers filled with conductive carbon black in thin films. With regard to nanoporous materials, studies have shown that the dielectric constant is overpredicted by the classical micromechanics approaches [12]. A possible explanation for such behaviour could be the simplified approximations of the local electric field at the void surface [13]. Lombardo [14] studied the electric properties of composites where spherical inclusions are embedded by an interphase. In analogy to the electric problem, effective thermal conductivities of composites accounting for interfaces have been studied e.g. in [15-19] and references therein. 
The aim of this research is to propose a micromechanics-based model for studying the electrostatic behaviour of spherical particle (or porous) composites when the size of the particle (or the pore) is in the range between nanometres and micrometres. The model identifies the effective dielectric constant (relative electric permittivity) of the composite, taking into account the independent behaviour of the interface between the particle and the matrix, in the spirit of the surface electrostatic theory presented in [20]. In contrast to the classical sphere's assemblage method, this manuscript takes the interface into account and therefore, the size effect is intrinsically inherited in this study. The paper is organized as follows: In Section 2, we present the general concepts of surface electricity; and in Section 3, we develop the micromechanics model, which is based on the composite spheres assemblage theory $[21,22]$. In Section 4, we present examples motivated from experimental results in porous media and particle composites and we compare the results obtained from our model with existing micromechanics theories. In the last section, we discuss the main conclusions of this work.

\section{Theory of surface electricity}

Here, we briefly summarize our work in [20], that recently presented a continuum mechanics framework for surface electrostatics. The results of the theory are valid for closed material interfaces, thus in the sequel we refer directly to composites with energetic interfaces. ${ }^{1}$ This approach does not simply consider an interface with zero thickness, but can rather be viewed as a simplification of an intrinsically nonlocal continuum theory whereby the interface captures the effective response of a thin interphase.

We consider a continuum body that occupies the open set $\mathscr{B} \subset \mathfrak{R}^{3}$ with boundary $\partial \mathscr{B}$. Inside the body there is a closed material interface, denoted by $\partial \mathscr{I}$, with normal vector $\boldsymbol{n}$. The electric fields $\mathbb{e}$ in the bulk and $\widehat{\mathbb{E}}$ on the interface are expressed in terms of a scalar potential $\varphi$ as

$$
\mathbb{e}=-\operatorname{grad} \varphi, \quad \widehat{\mathbb{e}}=-\widehat{\operatorname{grad}} \varphi,
$$

where we have assumed that the scalar potential is continuous across the interface. In the last expression, the surface gradient of an arbitrary vector $\{\bullet\}$ is given by

$$
\widehat{\operatorname{grad}}\{\bullet\}=\operatorname{grad}\{\bullet\} \cdot[\boldsymbol{i}-\boldsymbol{n} \otimes \boldsymbol{n}],
$$

and $\boldsymbol{i}$ is the second-order identity tensor. Variational principles allow us to express the electric displacement in the bulk, $\mathbb{d}$, and on the surface, $\widehat{\mathbb{d}}$, in terms of the internal potential energies in the bulk, $V^{\text {int }}(\mathbb{e})$, and on the surface, $\widehat{V}^{\text {int }}(\widehat{\mathbb{E}})$, respectively, as [20]

$$
\mathbb{d}=-\frac{\partial V^{\text {int }}}{\partial \mathbb{e}}, \quad \widehat{\mathbb{d}}=-\frac{\partial \widehat{V}^{\text {int }}}{\partial \widehat{\mathbb{e}}} .
$$

Under electrostatic conditions and ignoring any bulk and surface free charge density, the Maxwell equations read as

$$
\operatorname{curl} \mathbb{e}=\mathbf{0}, \quad \operatorname{div} \mathbb{d}=\mathbf{0},
$$

in the bulk and

$$
\boldsymbol{n} \times \llbracket \mathbb{\Perp} \rrbracket=\mathbf{0}, \quad \llbracket \mathbb{d} \rrbracket \cdot \boldsymbol{n}+\widehat{\operatorname{div} \widehat{\mathbb{d}}}=\mathbf{0},
$$


on the interface. The symbol $\llbracket\{\bullet\} \rrbracket$ denotes jump of a vector $\{\bullet\}$ across the interface, while the surface divergence is defined as

$$
\widehat{\operatorname{div}}\{\bullet\}=\widehat{\operatorname{grad}}\{\bullet\}:[\boldsymbol{i}-\boldsymbol{n} \otimes \boldsymbol{n}] .
$$

\section{Generalized self-consistent composite spheres assemblage for particle composites with electrically independent interfaces}

In order to compute the effective electrical behaviour of isotropic spherical particle composites with isotropic material interfaces we employ the generalized self-consistent composite spheres assemblage introduced by Christensen [22]. An analogous method has been proposed in the past [23] for studying the effective mechanical behaviour of particle composites with material interfaces, see also [18] for the thermal problem.

In this methodology, the actual composite (matrix and particle with material interface) is represented as an assemblage of three concentric spheres (Figure 1). The initial idealized representative volume element (particle with radius $r_{1}$ and matrix material between the radii $r_{1}$ and $r_{2}$ ) is connected with an external third layer which represents the effective medium and is extended to infinity $\left(r_{3} \rightarrow \infty\right)$. The interface at $r=r_{1}$ between the particle and the matrix is considered to have its own independent electric behaviour. Considering random distribution of particles inside the matrix, the isotropy of all constituents leads to isotropic behaviour for the effective medium. The constitutive laws of the materials in this case are written

$$
\mathbb{d}_{i}=-\varepsilon_{\mathrm{v}} \varepsilon_{i} \operatorname{grad} \varphi_{i} \quad \text { at } \quad r_{i-1} \leq r \leq r_{i} \quad \text { with } \quad r_{0}=0 .
$$

The dielectric constants (relative electric permittivity) are denoted by $\varepsilon_{i}$ and $\varepsilon_{\mathrm{v}}$ is the electric permittivity of vacuum. In a similar manner, the isotropic interface is characterized by a constitutive law of the form

$$
\widehat{\mathbb{d}}=-\varepsilon_{\mathrm{v}} \widehat{\varepsilon} \widehat{\operatorname{grad}} \varphi \quad \text { at } \quad r=r_{1} .
$$
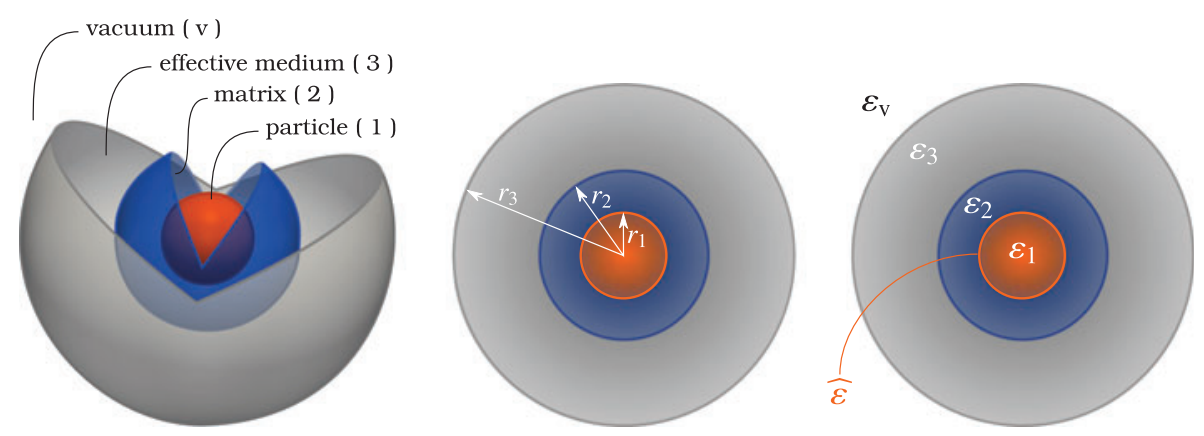

Figure 1. (colour online) Illustration of generalized self-consistent composite spheres assemblage for particle composites with electrically independent interfaces. The index $(i)$ denotes the material layer: $i=1$ for the particle, $i=2$ for the matrix and $i=3$ for the effective medium. $\varepsilon_{\mathrm{V}}$ denotes the electric permittivity of the vacuum (free space) and $\widehat{\varepsilon}$ the interface dielectric constant with the unit of length which leads to a more pronounced interface effect on the overall response of the body at small scales. Introducing an independent interfacial dielectric constant is the key feature of the current work. 
It is important to mention that, in contrast to the dimensionless dielectric constant $\varepsilon_{i}$, the interface material constant $\widehat{\varepsilon}$ must have unit of length.

In the sequel, all the equations will be expressed in spherical coordinates $(r, \theta, \phi)$. As boundary conditions we choose

$$
\varphi \rightarrow \beta r \cos \theta \quad \text { at } \quad r \rightarrow \infty .
$$

The sign of $\beta$ can be either positive or negative, since, as we show later, the overall dielectric constant is independent of $\beta$. Under these conditions, the Maxwell Equation ( 3$)^{2}$ for each material reduces to a two-dimensional problem in terms of $r$ and $\theta$,

$$
\frac{1}{r^{2}} \frac{\partial}{\partial r}\left(r^{2} \frac{\partial \varphi_{i}}{\partial r}\right)+\frac{1}{r^{2} \sin \theta} \frac{\partial}{\partial \theta}\left(\sin \theta \frac{\partial \varphi_{i}}{\partial \theta}\right)=0 \quad \text { at } \quad r_{i-1} \leq r \leq r_{i} .
$$

It can be shown that this equation has an analytical solution of the form

$$
\varphi_{i}=\left[A_{i} r+\frac{B_{i}}{r^{2}}\right] \cos \theta, \quad \text { at } \quad r_{i-1} \leq r \leq r_{i},
$$

where $A_{i}, B_{i}(i=1,2,3)$ are six constants. The computation of these six unknowns and of the dielectric constant of the effective medium, $\bar{\varepsilon}=\varepsilon_{3}$, is achieved with the following procedure:

The existence of a finite $\varphi$ at the centre of the particle $(r=0)$ leads to the conclusion that $B_{1}=0$. The boundary condition (7) defines the value of $A_{3}$ as $A_{3}=\beta$. For the rest of the constants and the effective relative permittivity we have the continuity conditions on the scalar potential

$$
\begin{array}{lll}
\varphi_{1}=\varphi_{2} & \text { at } & r=r_{1}, \\
\varphi_{2}=\varphi_{3} & \text { at } & r=r_{2},
\end{array}
$$

the jump condition (4), which in spherical coordinates is written

$$
-\varepsilon_{2} \frac{\partial \varphi_{2}}{\partial r}+\varepsilon_{1} \frac{\partial \varphi_{1}}{\partial r}-\frac{\widehat{\varepsilon}}{r_{1}^{2}}\left[\frac{\partial^{2} \varphi_{1}}{\partial \theta^{2}}+\frac{\partial \varphi_{1}}{\partial \theta} \frac{\cos \theta}{\sin \theta}\right] \text { at } r=r_{1},
$$

and the electric flux continuity condition on the interface between the matrix and the effective medium

$$
-\varepsilon_{3} \frac{\partial \varphi_{3}}{\partial r}+\varepsilon_{2} \frac{\partial \varphi_{2}}{\partial r}=0 \quad \text { at } \quad r=r_{2}
$$

The last necessary equation is derived from a similar energy principle with the one that is expressing the mechanical problem in the composite spheres assemblage [see [22], Equation (4.10) on p.56]. This last condition leads to the simple result $B_{3}=0$. Thus, from the system of equations $(10)_{1,2}-(12)$ we compute the effective relative permittivity

$$
\bar{\varepsilon}=\varepsilon_{2}\left[1+\frac{f\left[1+2 \frac{\widehat{\varepsilon} / r_{1}}{\varepsilon_{1}-\varepsilon_{2}}\right]}{\frac{1-f}{3}\left[1+2 \frac{\widehat{\varepsilon} / r_{1}}{\varepsilon_{1}-\varepsilon_{2}}\right]+\frac{\varepsilon_{2}}{\varepsilon_{1}-\varepsilon_{2}}}\right],
$$

where $f=\left[r_{1} / r_{2}\right]^{3}$ is the volume fraction of the particles in the matrix. 

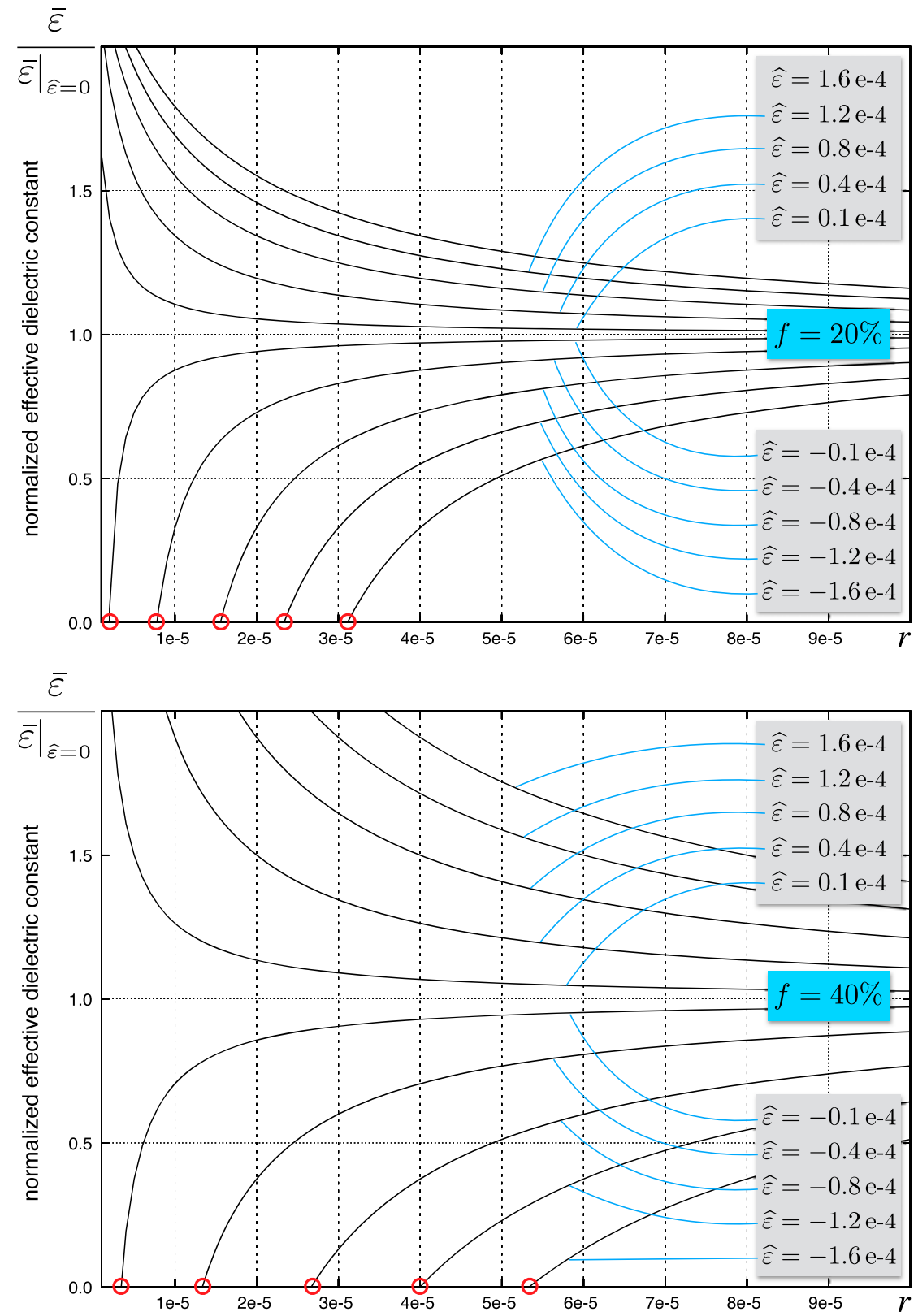

Figure 2. (colour online) Effective dielectric constant versus void radius for various surface dielectric constants $\widehat{\varepsilon}$ for two volume fractions of $f=20 \%$ and $40 \%$. The effective dielectric constant is calculated from the analytical solution (13) assuming $\varepsilon_{1}=1$ and $\varepsilon_{2}=10$ and is normalized with the effective dielectric constant corresponding to $\widehat{\varepsilon}=0$. Therefore, the value 1 on the vertical axis indicates the effective response using classical homogenization and clearly, missing the size effect. Taking the surface dielectric constants $\widehat{\varepsilon}$ into account, introduces a size effect. Positive values for the surface dielectric constant lead to an increasing effective response for decreasing size. Negative surface dielectric constant shows an opposite behaviour. Interestingly, for $\widehat{\varepsilon}<0$ there will be a critical size at which the effective dielectric constant vanishes indicated red circles on the graphs. 
Figure 2 provides a parametric study to better understand the behaviour of the effective response (13) versus the void radius or equivalently, the characteristic length of the microstructure. The graphs pertain to a porous material with $\varepsilon_{1}=1$ indicating vacuum response for the voids and $\varepsilon_{2}=10$ is arbitrarily chosen for the matrix motivated by the fact that the relative permittivity for most materials exceeds that of the vacuum. This study is carried out for two different volume fractions of $f=20 \%$ and $40 \%$. The vertical axis on Figure 2 represents the normalized effective dielectric constant. The effective dielectric constant is normalized by its value corresponding to $\widehat{\varepsilon}=0$. Therefore, the value 1 on the vertical axis indicates the effective dielectric constant of the porous material when surface effects are neglected, i.e. the effective response corresponding to classical homogenization. Note, size effect can be only observed if the surface effects are taken into account.

Positive values for the surface dielectric constant lead to an increasing effective response for decreasing size leading to a "smaller is stronger" behaviour. In contrast, a "smaller is weaker" response is obtained for negative surface dielectric constant. However, the surface dielectric constant cannot be arbitrarily small when it assumes negative values since stability analysis requires the effective dielectric constant to be positive. That is, for a given microstructure there exists a critical value of the surface dielectric constant. In other words, for any negative surface dielectric constant there exists a critical microstructure size where the overall dielectric constant vanishes. These critical values are indicated by red circles on the graphs of Figure 2.

\section{Examples}

In this section, we study certain cases from the literature in which the dielectric constant of composites has been obtained from experimental tests. Our intention is to simulate the composites behaviour using our proposed micromechanics framework and also to compare our results with other available models, see [24] for a review of available models.

\subsection{Dielectric constant of porous media}

In Figure 3, we present experimental results for two different porous media, sintered alumina [25] and nanofoam samples [12]. Apart from the Voigt upper and Reuss lower bounds, we can find in the literature certain micromechanics models for estimating the dielectric constant of porous media, like in [26-28]. In all these models, it is assumed that the pores have spherical shape and dielectric constant equal to 1 . We also note that the Maxwell-Garnett method provides exactly the same solution as the classical composite spheres assemblage method without independent interface response.

(1) In the case of sintered alumina [25] the authors do not report specific pore size, but they have studied sintered alumina with grain size between 1 and $7 \mu \mathrm{m}$. The dielectric constant of almost nonporous polycrystalline alumina is close to 10 . In Figure 3(a), we observe that the classical micromechanics techniques predict quite well the experimental results and the best behaviour is observed for the Heidinger and Nazare model. Our micromechanics method with interface (current model) presents good agreement with the experimental results for $\widehat{\varepsilon} / r_{1}=-0.45$.

(2) In the case of nanofoams [12] the authors mention that the produced pores are in the nanometre dimension regime and the polymer matrix dielectric constant is close 

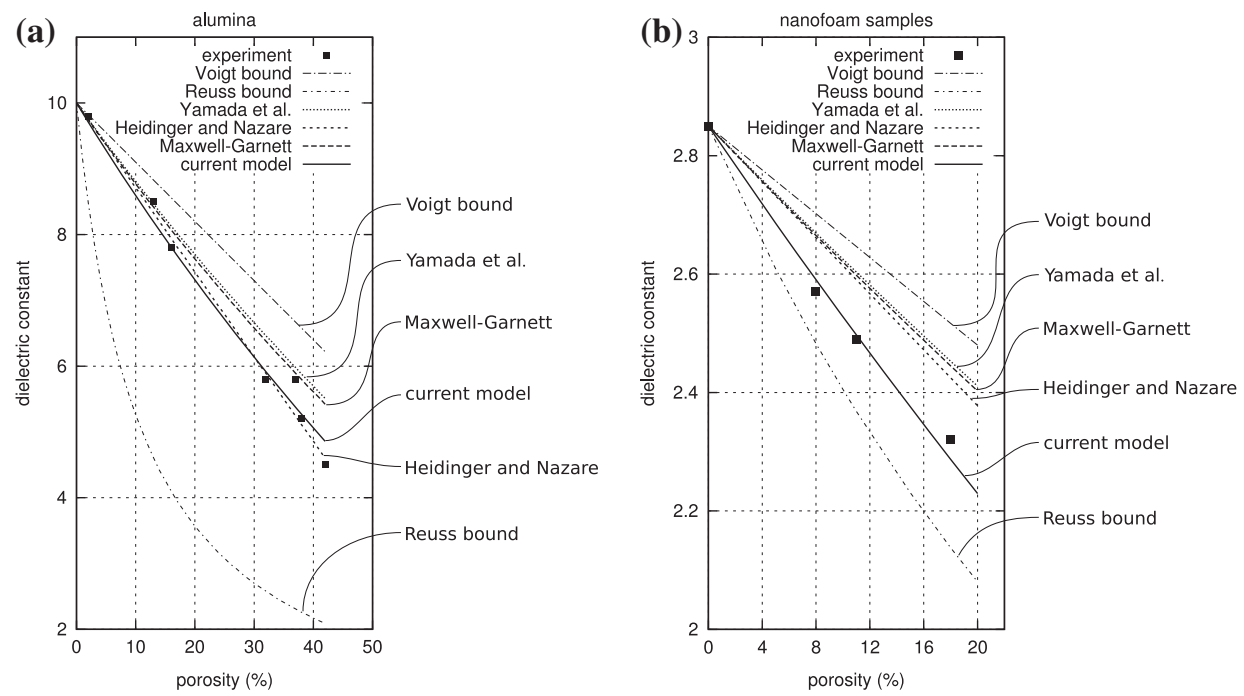

Figure 3. Effective dielectric constant versus porosity for (a) alumina [25] and (b) nanofoam samples [12].

to 2.85. As it is illustrated in Figure 3(b), the classical micromechanics approaches overpredict the electrical behaviour of the nanofoam samples and this can be due to local effects in the surface of the pores. In our simulation (current model) the obtained effective response agrees quite well with the experimental observations for $\widehat{\varepsilon} / r_{1}=-0.2775$.

\subsection{Dielectric constant of $\mathrm{BaTiO}_{3}$ particle composites}

In Figure 4, we present experimental results for barium titanate particle composites [7,29,30] and their simulations using different micromechanics models. Apart from the current model, we also present results obtained using the theories of [26,27,31]. All the models consider that the particles have spherical shape and they are applicable for electrostatic conditions, thus all the examined experimental cases are conducted under low frequency. With regard to the barium titanate, it has been experimentally observed that the grain size influences its electrical behaviour [32].

(1) In the first case of Figure 4 [29], the examined barium titanate powder has grain size of approximately $1 \mu \mathrm{m}$. The authors report the dielectric constants of the particles (barium titanate) and the matrix (polyvinylidene fluoride [PVDF]) to be 3,000 and 8.5 , respectively. The performed tests indicate that for barium titanate volume fraction above $50 \%$ the composite dielectric constant decreases due to pores caused by the high concentration of ceramic fillers, and thus we do not consider these cases in the present analysis. Figure 4(a) shows that the Jayasundere and Smith model predicts quite well the composite's behaviour. Our model has good agreement with the experimental results when we consider $\widehat{\varepsilon}=-768.6 \mu \mathrm{m}$.

(2) In the second case of Figure 4 [30], the barium titanate powder produced by TAM Ceramics Inc. has grain size of approximately $1 \mu \mathrm{m}$. The authors consider that the 
(a) $\mathrm{BT} / \mathrm{PVDF}$ (BT grain size $1 \mu \mathrm{m}$ )

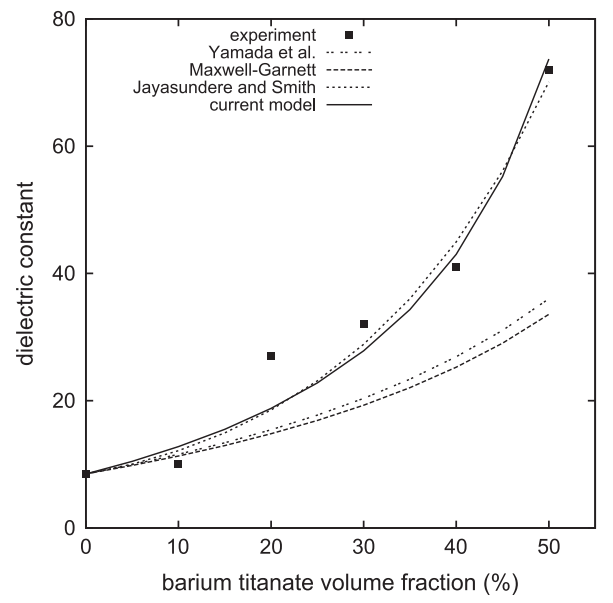

(c) SS-BT/epoxy (BT grain size $1 \mu \mathrm{m}$ )

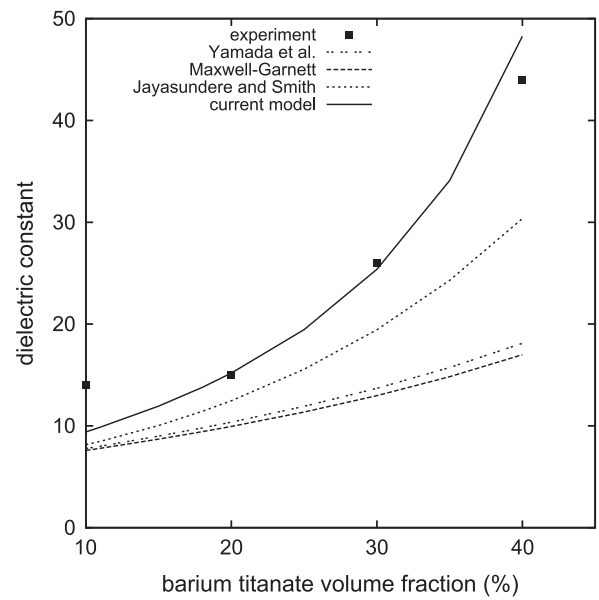

(b) TAM-BT/epoxy (BT grain size 1 $\mu \mathrm{m})$

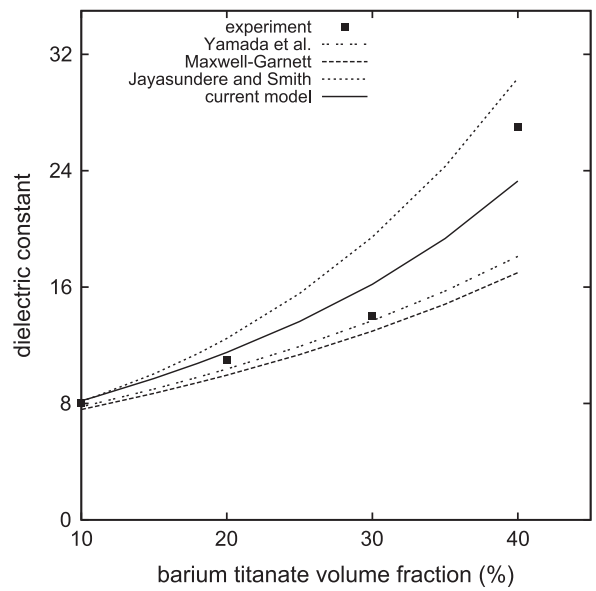

(d) BT/PVDF (BT particle size 100 $\mathrm{nm}$ )

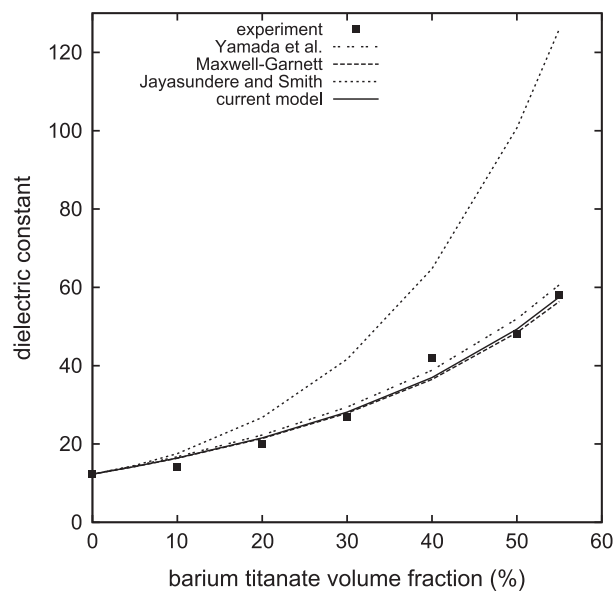

Figure 4. Composites with barium titanate (BT) particles: (a) experiments conducted by [29] using PVDF as matrix, (b) experiments conducted by [30] using epoxy as matrix and $\mathrm{BaTiO}_{3}$ powder produced by TAM Ceramics Inc., (c) experiments conducted by [30] using epoxy as matrix and selfsynthesized $\mathrm{BaTiO}_{3}$ powder, (d) experiments conducted by [7] using PVDF as matrix and untreated barium titanate particles.

dielectric constant of the epoxy matrix is equal to 5.7. Kuo et al. [30] mention that for grain size of $1 \mu \mathrm{m}$ the barium titanate powder presents maximum dielectric constant of 5,000-6,000. From their parametric analysis, the authors show that for particle dielectric constant above 1,000, the effective response does not present significant change. For comparison reasons with the case of Figure 4(a), in the current analysis we assume that the dielectric constant of the barium titanate powder is 3,000 and we also choose for our model the surface dielectric constant to be $\widehat{\varepsilon}=-768.6 \mu \mathrm{m}$. As 
it is observed in Figure 4(b), our model has better agreement with the experimental results compared to the other methods.

(3) In the third case of Figure 4 [30], the barium titanate powder is self-synthesized and has grain size of approximately $1 \mu \mathrm{m}$. The authors use the same matrix properties as in the previous case (dielectric constant equal to 5.7). Since for particle dielectric constant above 1,000 the effective response does not present significant change, we assume that the barium titanate powder has dielectric constant 3,000 and the experimentally different results between the second and the third case are due to different surface behaviour between the self-synthesized and the TAM-BT powder. In this case, we choose for our model the surface dielectric constant to be $\widehat{\varepsilon}=$ $-758.3 \mu \mathrm{m}$ and we observe that we can capture quite well the experimental data, while the other models underpredict the effective dielectric constant (Figure 4(c)).

(4) In the fourth case of Figure 4 [7], the barium titanate particles have an average size of about $100 \mathrm{~nm}$. The authors report the dielectric constants of the particles (barium titanate) and the matrix (PVDF) to be 3,500 and 12.3, respectively. The experimental results for $60 \%$ volume fraction have been excluded, since the models do not account for agglomerations and air voids. We choose for our model the surface dielectric constant to be equal to one for the cases 1 and $2(\widehat{\varepsilon}=-768.6 \mu \mathrm{m})$, thus we assume that the change in particles size and electric behaviour does not influence the surface properties. In this case, most of the micromechanical models and the one we propose capture quite well the experimental data (Figure 4(d)).

The four examined cases in this subsection indicate that the surface dielectric constant for particle composite seems to depend only on the particle and not on the matrix.

Remark (Negative interface dielectric constant is admissible) In the present model, negative values for the dielectric constant are admissible, in general. From a mathematical point of view, the admissible range for the interface dielectric constants could be calculated from Equation (13) by imposing overall response to be positive. The negative dielectric constant shall be compared to the negative magnetic permeability in diamagnetic materials, for instance. Furthermore, we recall the analogy of this problem to the well-established surface elasticity theory [33-36] in the mechanical case. It is known that the admissible range for the surface material parameters can differ from those in the bulk. In particular, surface material parameters can be negative where the analogous bulk material parameters are strictly positive. This can be justified by the fact that the surface cannot exist independent of the bulk and the overall response of the bulk together with the surface need to be positive definite [37-39].

\section{Conclusion}

In order to study the electrostatic behaviour of composites and porous materials, a micromechanical model is proposed that endows the interface between the particle (or the pore) and the matrix with its own independent behaviour. The model employs the wellestablished composite spheres assemblage method and enhances the interface with its own dielectric constant. It is shown that the current model has an excellent agreement with experiments and embraces better capabilities to predict realistic material behaviour. It is also observed that using different experimental data leads to the same interface dielectric 
constant depending only on the particle and not the matrix. Finally, it shall be mentioned that in all the examined cases the current model simulated quite well the experimental results for one interface dielectric constant.

\section{Acknowledgements}

The support of this work by the ERC Advanced Grant MOCOPOLY is gratefully acknowledged. The first author would like to acknowledge the support by the King Abdullah University of Science and Technology (KAUST) Center for Numerical Porous Media.

\section{Disclosure statement}

No potential conflict of interest was reported by the authors.

\section{Note}

1. The label energetic denotes that the interface possesses electrical and constitutive structures. These structures are independent from those of the bulk.

\section{References}

[1] M.G. Todd and F.G. Shi, J. Appl. Phys. 94 (2003) p.4551.

[2] M. Roy, J.K. Nelson, R.K. MacCrone, L.S. Schadler, C.W. Reed, R. Keefe and W. Zenger, IEEE Trans. Dielectr. Electr. Insul. 12 (2005) p.629.

[3] B. Kchaou, C. Turki, M. Salvia, Z. Fakhfakh and D. Trheux, Compos. Sci. Technol. 64 (2004) p.1467.

[4] K.S. Park, J.T. Son, H.T. Chung, S.J. Kim, C.H. Lee, K.T. Kang and H.G. Kim, Solid State Commun. 129 (2004) p.311.

[5] D. Ma, T.A. Hugener, R.W. Siegel, A. Christerson, E. Mårtensson, C. Önneby and L.S. Schadler, Nanotechnology 16 (2005) p.724.

[6] T. Wang, W. Li, L. Luo and Y. Zhu, Appl. Phys. Lett. 102 (2013) p.092904.

[7] K. Yu, Y. Niu, Y. Zhou, Y. Bai and H. Wang, J. Am. Ceram. Soc. 96 (2013) p.2519.

[8] H. Lee and H. Kim, J. Appl. Phys. 67 (1990) p.2024.

[9] P. Papet, J.P. Dougherty and T.R. Shrout, J. Mater. Res. 5 (1990) p.2902.

[10] D.N. McCarthy, H. Stoyanov, D. Rychkov, H. Ragusch, M. Melzer and G. Kofod, Compos. Sci. Technol. 72 (2012) p.731.

[11] K. Wongtimnoi, B. Guiffard, A.B.V. de Moortle, L. Seveyrat, C. Gauthier and J.Y. Cavaill, Compos. Sci. Technol. 71 (2011) p.885.

[12] J.L. Hedrick, R.D. Miller, C.J. Hawker, K.R. Carter, W. Volksen, D.Y. Yoon and M. Trollsås, Adv. Mater. 10 (1998) p.1049.

[13] C.K. Min, T.B. Wu, W.T. Yang and C.L. Chen, Compos. Sci. Technol. 68 (2008) p.1570.

[14] N. Lombardo, Compos. Sci. Technol. 67 (2007) p.728.

[15] Y. Benveniste, J. Mech. Phys. Solids 54 (2006) p.708.

[16] H. Duan and B. Karihaloo, Phys. Rev. B 75 (2007) p.064206.

[17] J. Yvonnet, Q.C. He and C. Toulemonde, Compos. Sci. Technol. 68 (2008) p.2818.

[18] H. Le-Quang, G. Bonnet and Q.C. He, Phys. Rev. B 81 (2010) p.064203.

[19] A. Javili, S. Kaessmair and P. Steinmann, Comput. Methods Appl. Mech. Eng. 275 (2014) p.76.

[20] G. Chatzigeorgiou, A. Javili and P. Steinmann, Proc. R. Soc. A 470 (2014) p.20130628.

[21] Z. Hashin and S. Shtrikman, J. Appl. Phys. 33 (1962) p.3125. 
[22] R.M. Christensen, Mechanics of Composite Materials, New York: Dover, 1979.

[23] H.L. Duan, J. Wang, Z.P. Huang and B.L. Karihaloo, J. Mech. Phys. Solids 53 (2005) p.1574.

[24] M.C. Araujo, C. Costa and S. Lanceros-Mendez, J. Non-Cryst. Solids 387 (2014) p.6.

[25] S.J. Penn, N.M. Alford, A. Templeton, X. Wang, M. Xu, M. Reece and K. Schrapel, J. Am. Ceram. Soc. 80 (1997) p.1885.

[26] J.C. Maxwell-Garnett, Phil. Trans. R. Soc. A 23 (1904) p.385.

[27] T. Yamada, T. Ueda and T. Kitayama, J. Appl. Phys. 53 (1982) p.4328.

[28] R. Heidinger and S. Nazare, Powder Metall. Int. 20 (1988) p.30.

[29] Z.M. Dang, Y. Shen and C.W. Nan, Appl. Phys. Lett. 81 (2002) p.4814.

[30] D.H. Kuo, C.C. Chang, T.Y. Su, W.K. Wang and B.Y. Lin, Mater. Chem. Phys. 85 (2004) p.201.

[31] N. Jayasundere and B.V. Smith, J. Appl. Phys. 73 (1993) p.2462.

[32] K. Kinoshita and A. Yamaji, J. Appl. Phys. 47 (1976) p.371.

[33] M.E. Gurtin and A.I. Murdoch, Arch. Ration. Mech. Anal. 57 (1975) p.291.

[34] A. Javili and P. Steinmann, Comput. Methods Appl. Mech. Eng. 198 (2009) p.2198.

[35] A. Javili and P. Steinmann, Comput. Methods Appl. Mech. Eng. 199 (2010) p.755.

[36] A. Javili, A. McBride, P. Steinmann and B.D. Reddy, Comput. Mech. 54 (2014) p.745.

[37] V.B. Shenoy, Phys. Rev. B 71 (2005) p.094104.

[38] A. Javili, A. McBride, P. Steinmann and B.D. Reddy, Phil. Mag. 92 (2012) p.3540.

[39] A. Javili, F. dell'Isola and P. Steinmann, J. Mech. Phys. Solids 61 (2013) p.2381. 